\title{
La especificidad del desarrollo de la industria automotriz en la Argentina, 1959-1963
}

VALERIA L. IANNI*

Resumo: A forma específica em que a indústria automobilística se desenvolveu na Argentina, a partir de 1959 (crescimento e decréscimo de empresas, produção em pequena escala, produtividade abaixo da média mundial, entre outros), é revisada neste artigo, a partir de uma perspectiva que centra a explicação na lógica do movimento do capital a nível internacional, no período posterior à Segunda Guerra Mundial.

Abstract: The specific way in which the automobile industry has developed in Argentina since 1959 (increase and decrease in the number of companies, smallscale production, productivity below the world average, among others) is revised in this work from a perspective that centres the explanation around the logic of the movement of capital at international level in the second postwar period.

Palavras-chave: Argentina. Indústria automobilística. Lógica do capital.

Key words: Argentina. Automotive industry. Logic of capital.

\section{Introducción}

La instalación y desarrollo de las empresas terminales transnacionales que protagonizaron la fuerte expansión de la rama automotriz en Argentina, con peculiar ímpetu desde fines de la década de 1950 y los comienzos de la siguiente, fue uno de los aspectos más salientes de la "fase difícil" de la denominada "industrialización por sustitución de importaciones" (ISI).

* Doctoranda de Historia en la Facultad de Filosofía y Letras de la Universidad de Buenos Aires. Becaria del CONICET con asiento en el Instituto de Historia Argentina y Latinoamericana "Dr. Emilio Ravignani", UBA. Integrante del Proyecto UBACyT E 046.

Estudos Ibero-Americanos, PUCRS, v. XXXIV, n. 2, p. 97-113, dezembro 2008 
A pesar de la rapidez con que esta rama se consolidó dentro de la estructura industrial, de su innegable efecto multiplicador sobre otros sectores y subsectores, y de su contribución a la transformación de la capacidad tecnológica de la sociedad en su conjunto, muy tempranamente se revelaron con nitidez las limitaciones que enfrentaba la rama en el país.

El presente trabajo se inserta dentro de un conjunto de investigaciones que a lo largo de varias décadas se han preguntado acerca de las razones que explican esta particularidad del desarrollo de esa industria que a nivel mundial fue el símbolo del éxito capitalista de la segunda posguerra. La hipótesis que buscaremos demostrar es que esta especificidad obedeció fundamentalmente al carácter contradictorio de la expansión de la acumulación de capital que, por un lado, extendió a países como Argentina la producción de "bienes de consumo durable", pero que, al hacerlo, reprodujo el desarrollo desigual en lugar de producir una inmediata homogeneización de las condiciones de producción.

\section{El "dinamismo" de la industria automotriz}

La preocupación acerca de las condiciones en las que se expandió la producción automotriz en Argentina dio lugar a una serie de trabajos específicos. Asimismo, los estudios que se centran en la historia económica e industrial han dedicado importantes fragmentos a este caso. ${ }^{1}$

De acuerdo a datos del Censo Nacional Económico de 1964, la división 38301 (Fabricación y armado de vehículos automóviles completos: automóviles, camiones, camionetas, y demás vehículos análogos) con un total de 17 establecimientos ocupaba a 24.470 personas de las cuales 17.643 eran obreros,

1 La presentación más completa continúa siendo la de Sourrouille (1980). El libro de Baranson (1971) cuenta con una investigación de campo sobre Argentina. En Brennan (1996) hay un profundo análisis de las empresas automotrices instaladas en Córdoba. A pesar de centrarse en períodos disímiles, tanto Schvarzer (1995) como Belini (2006) contribuyen a una visión más global de la rama. Por su parte, Catalan (2007) ha presentado un trabajo comparativo entre esa industria en Argentina, Corea y España. Desde la producción de organismos estatales se destacan para el período bajo análisis CONADE (1966) y Coscia (1980). En cuanto a la extensa historiografía de la industria que se dedica al sector automotriz, remitimos centralmente a Altimir et al. (1966/67); Dorfman (1983), Katz y Kosacoff (1989); y Schvarzer (1987 y 2000). 
cifra que representaría aproximadamente el 2\% del total de ocupación para el país, calculada generosamente en 1.370 .486 personas. $^{2}$ Sin embargo, hay que tomar en cuenta el importante efecto que tuvo la instalación de terminales automotrices en la activación de un conjunto sumamente variado de ramas y subramas proveedoras de materias primas, de partes y conjuntos de partes, además de la gran cantidad de personas empleadas en tareas de reparación. Sourrouille (1980, cuadro 6) calcula que la incidencia de todo el complejo automotor en la ocupación rondaba el 10\%. De una mirada diacrónica, surge que la cantidad de obreros ocupados en la rama, entre 1959 y 1965, se multiplicó casi por cuatro (de 8.127 a 23.961), destacándose que la proporción de operarios calificados supera a la de peones (CONADE, 1966, p. 34 y cuadro 23).

En relación con el total nacional del valor agregado industrial, sólo el subgrupo de fabricación y armado de la industria automotriz aportaba el 4,5\% (Sourrouille, 1980, cuadro 6). Por otra parte, la rama fue uno de los destinos principales de las inversiones extranjeras, recibiendo casi la cuarta parte del total de las autorizaciones para radicación en el período 1959-1962 (Schvarzer, 1987, p. 34). La tasa media anual de inversiones en el sector fue entre 1960 y 1964 casi diez veces superior a la del período 1956-1959 (Baranson, 1971, p. 57). Por otra parte, entre 1958 y 1965 la industria automotriz, con 32,3\% del total, fue el sector con mayor participación en el aumento del producto industrial (Heymann citado en Jerez, 2007, p. 35). La tasa anual acumulativa de crecimiento del sector automotriz fue en ese mismo lapso de tiempo, de 11,16\% (Coscia, 1980, II.3).

Como consecuencia de estas transformaciones, no resulta llamativo que la relación entre población total y automóviles no comerciales se redujera a la mitad. En 1959 este índice era de 47,8 habitantes por automóvil y seis años después era de 23,7 (Coscia, 1980, cuadro II.2).

2 Hay que destacar ciertos problemas que presenta esta fuente. La cantidad de establecimientos, relevada el 30 de mayo de 1964, no se corresponde con la que se recaba en la bibliografía. El criterio empleado fue sumamente amplio: "El censo manufacturero tomó todos los establecimientos que a la fecha del relevamiento estaban activos o no, sin distinción de magnitud o importancia de los mismos" (INDEC, 1965 , p. 5, destacado nuestro). Si agregamos a esto el hiato entre lo declarado y lo real, concluimos que las cifras no son una evidencia irrefutable sino un índice de tendencias generales (Schvarzer, 1977). 


\section{La historiografía sobre la industria automotriz en Argentina}

Los datos del apartado anterior considerados aisladamente parecerían apuntar a un desempeño altamente exitoso de la industria automotriz. Sin embargo, esto no fue así. Las empresas automotrices instaladas localmente no emplearon la tecnología que imperaba en la frontera internacional, tampoco fomentaron la innovación. Por el contrario, la rama operó con un alto grado de obsolescencia. Tampoco se concretó el alivio en el balance de pagos que se suponía vendría de la mano del avance en la sustitución de importaciones.

Si el capital en su internacionalización homogeneizara de un modo inmediato las condiciones de producción, el problema que planteamos - cómo dar cuenta del desarrollo de una rama particular, en nuestro caso la automotriz, en una formación social determinada como la Argentina - carecería de importancia. Tampoco sería pertinente hablar de "especificidad" si la diferencia existente entre la producción automotriz local e internacional obedeciera a meras divergencias cuantitativas originadas en la diferencia de estadíos en el despliegue de la rama. En ese caso, sería comprensible que una conformación tardía de la industria trajera aparejada una distancia inicial respecto de las economías en las que el sector automotriz ya había alcanzado una etapa de madurez.

Sin embargo, la realidad de la industria automotriz en sus años iniciales muestra que existió una relación mucho más compleja y contradictoria. Por un lado, la industria local pudo "quemar" ciertas etapas que ya había atravesado en los países más industrializados. Pero por otro, reprodujo todas las limitaciones ligadas a la pequeña escala de la producción local. ${ }^{3}$ Este problema de cómo dar cuenta de estos dos aspectos de la realidad es el que ha atravesado las investigaciones sobre este caso.

En líneas generales, los autores han destacado el origen de la rama como una de las claves para entender este desarrollo

3 "El sector de capital extranjero se caracteriza: a) porque en comparación con las firmas competidoras de capital nacional, son mayores sus tamaños de planta, la productividad de su mano de obra, sus coeficientes de importación y su dotación de capital por hombre empleado; b) porque su comportamiento tecnológico se basa en la incorporación de tecnologías que, a pesar de no ser de frontera a escala internacional, sin duda son novedosas en el mercado local" (Katz y Kosacoff, 1989, p. 29, destacado nuestro). 
particular. ${ }^{4}$ Especialmente, han destacado la influencia que tuvo la legislación que regulaba a la industria automotriz en la forma en que ésta se estructuró. Además de las disposiciones generales de la Ley 14.780 sobre inversión de capitales extranjeros y la 14.781 de promoción industrial (Anales de Legislación Argentina, 1958, tomo XVIIIA, p. 314-317), la automotriz contó desde la sanción del decreto $3693 / 59$, con un régimen de promoción sectorial (ALA, 1959, tomo XIX A, p. 617-619). Éste exigía la demostración de cierta capacidad técnica y financiera, la progresiva integración nacional del producto para reducir importaciones, además de la presentación de planes de producción de 5 años. ${ }^{5}$ Para fomentar el desarrollo de esta industria, el estado elevaba a niveles prohibitivos la importación de automóviles terminados (Altimir et al., 1966, p. 101).

Las investigaciones coinciden en criticar fuertemente ese modelo de promoción al sector automotriz por su laxitud en la aprobación de inversiones que condujo a una expansión del número de empresas completamente desmedido respecto del tamaño del mercado local y a altos niveles de ineficiencia. ${ }^{6}$ Como no podía ser de otro modo, muchas de las empresas que habían presentado sus planes de producción desaparecieron del mercado a los pocos años, mientras que algunas ni siquiera llegaron a iniciar la producción.

Desde la perspectiva teórico-metodológica que adoptamos, compartimos la caracterización general de la rama que han llevado a cabo diversos estudiosos, pero discrepamos en la identificación de las causas que condujeron a ese resultado. No obstante, consideramos que la perspectiva institucionalista sobreestima la capacidad de las políticas económicas para dar forma a la economía real. Sin negar la importancia que tiene la interacción entre ambas esferas, sostenemos que la razón de ese desarrollo específico se encuentra en la lógica contradictoria del capital. Intentaremos demostrar esto revisando ciertos rasgos cruciales de la industria

4 Belini (2006) ha criticado el consenso historiográfico que relega a las experiencias previas a 1959 al papel de "antecedente". A pesar de las dificultades que implica cortar un proceso de desarrollo global, creemos necesario poder encontrar los momentos en que un salto cualitativo modifica el proceso. En este sentido, resulta indudable que el año 1959 marca el inicio de una nueva fase para el sector.

5 El primer quinquenio era en la práctica más extenso ya que en el decreto estableció que "se considerará como fecha de vencimiento del primer año de los planes de fabricación el 31 de diciembre de 1960".

6 Con matices, esta lectura se encuentra en: CONADE, 1963, aut1; CONADE, 1965, p. 56; Katz y Kosacoff, 1989, p. 55-56; Schvarzer, 2000, p. 226; Dorfman, 1983, p. 196; Belini, 2006, p. 130; Baranson, 1971, p. 64; Sourrouille: 1980, p. 22. 
automotriz en el momento de su mayor expansión en cuanto a número de empresas en 1959 hasta el ajuste violento que se dio con la crisis general de la economía argentina en 1962-1963, y que en la rama bajo estudio se hizo sentir con fuerza en los primeros ocho meses del segundo de esos años (ADEFA, 1964, p. 4).

\section{La depuración de capitales tras la expansión}

En el Cuadro 1 presentamos la lista completa de las empresas que presentaron planes de producción para acogerse a los beneficios del Decreto 3693/59, el año en que iniciaron el programa de producción (si es que lo hicieron) y en el que se retiraron de la actividad. ${ }^{7}$

CUADRO 1

Empresas autorizadas a producir bajo el Régimen Automotor

Continua

\begin{tabular}{|c|c|c|c|}
\hline Empresa & $\begin{array}{l}\text { Año de } \\
\text { inicio }\end{array}$ & $\begin{array}{l}\text { Año de } \\
\text { cierre }\end{array}$ & Observaciones \\
\hline Alcre S.A. & 1959 & 1961 & $\begin{array}{l}\text { No alcanzó a producir } \\
\text { (sólo presentó prototipos) }\end{array}$ \\
\hline $\begin{array}{l}\text { Autoar Automotores } \\
\text { Argentinos S.A. }\end{array}$ & 1949 & 1963 & $\begin{array}{l}\text { En } 1964 \text { sólo produjo } 10 \\
\text { vehículos. }\end{array}$ \\
\hline Cisitalia Argentina S.A. & 1961 & 1963 & \\
\hline Citröen Argentina S.A. & 1960 & & \\
\hline Dinámica Argentina Dinarg & - & - & $\begin{array}{l}\text { No hay datos de que haya } \\
\text { producido. }\end{array}$ \\
\hline $\begin{array}{l}\text { Dinborg - Industrias Argentinas } \\
\text { Automotrices }\end{array}$ & 1958 & 1963 & \\
\hline $\begin{array}{l}\text { Fábrica de Automotores } \\
\text { Utilitarios S.A. }\end{array}$ & 1960 & 1963 & \\
\hline $\begin{array}{l}\text { Févre y Basset Ltda., S.A. y } \\
\text { Chrysler Argentina S.A. }\end{array}$ & 1961 & & $\begin{array}{l}\text { Desde } 1960 \text { producía } \\
\text { utilitarios, su plan de } \\
\text { producción de automóviles } \\
\text { fue aprobado en } 1961\end{array}$ \\
\hline Fiat Someca Concord S.A. & 1960 & & $\begin{array}{l}\text { Desde } 1954 \text { producía } \\
\text { tractores en el país }\end{array}$ \\
\hline Ford Motor Argentina S.A. & 1961 & & $\begin{array}{l}\text { Desde } 1959 \text { producía } \\
\text { utilitarios, su plan de } \\
\text { producción de automóviles } \\
\text { fue aprobado en } 1961\end{array}$ \\
\hline
\end{tabular}

7 Varias de las empresas que están en el Cuadro 1 dejaron de existir luego de 1965. No incluimos esos datos para resaltar los cambios ocurridos bajo el período delimitado. 


\section{CUADRO 1}

Empresas autorizadas a producir bajo el Régimen Automotor

Conclusión

\begin{tabular}{|c|c|c|c|}
\hline Empresa & $\begin{array}{c}\text { Año de } \\
\text { inicio }\end{array}$ & $\begin{array}{l}\text { Año de } \\
\text { cierre }\end{array}$ & Observaciones \\
\hline General Motors Argentina S.A. & 1961 & & $\begin{array}{l}\text { Desde } 1959 \text { producía } \\
\text { utilitarios, su plan de } \\
\text { producción de automóviles } \\
\text { fue aprobado en } 1961\end{array}$ \\
\hline Goliath Hansa Argentina S.A. & 1960 & 1961 & \\
\hline IAFA S.A. (Peugeot) & 1960 & 1964 & $\begin{array}{l}\text { Se convirtió en SAFRAR a } \\
\text { raíz de un problema legal. }\end{array}$ \\
\hline D.I.N.F.I.A. (ex I.A.M.E.) & 1953 & & \\
\hline Imena S.A. & - & - & $\begin{array}{l}\text { No hay datos de que haya } \\
\text { producido }\end{array}$ \\
\hline $\begin{array}{l}\text { Industria Automotriz } \\
\text { Santa Fe S.A. }\end{array}$ & 1960 & & \\
\hline $\begin{array}{l}\text { Industrias de Transporte } \\
\text { Automotor S.A. }\end{array}$ & 1960 & 1961 & \\
\hline Industrias Kaiser Argentina S.A. & 1956 & & \\
\hline $\begin{array}{l}\text { Industrias Platenses } \\
\text { Automotrices }\end{array}$ & - & - & $\begin{array}{l}\text { No hay datos de que haya } \\
\text { producido }\end{array}$ \\
\hline Isard Argentina S.A. & 1960 & & \\
\hline Los cedros S.A. & 1957 & 1962 & $\begin{array}{l}\text { En } 1963 \text { se fusionó con } \\
\text { Isard, adoptando el nombre } \\
\text { de esta última. }\end{array}$ \\
\hline Mercedes Benz Argentina S.A. & 1959 & & $\begin{array}{l}\text { En } 1953 \text { había iniciado la } \\
\text { fabricación en el país, pero } \\
\text { fue interdicta por el golpe } \\
\text { de } 1955 .\end{array}$ \\
\hline Metalmecánica S.A. & 1958 & & \\
\hline Onofre Marimón y Cía. SRL & 1960 & 1961 & \\
\hline Panambí S.A. & 1960 & 1961 & \\
\hline Siam Di Tella Automotores S.A. & 1960 & & \\
\hline
\end{tabular}

FUENTE: elaboración propia en base a CONADE, 1966, Anexo A, cuadros 31 y 32, y Sourrouille, 1980.

A partir de los datos del Cuadro 1, podemos afirmar que el período bajo análisis puede dividirse a su vez en dos momentos, ambos muy breves para el tiempo que implica una inversión fabril. El primero, de alrededor de dos años, se extiende hasta el primer saneamiento de la cantidad de empresas. No hablamos de fábricas sino de empresas debido a que muchas de ellas eran simples talleres. Acordamos con Schvarzer $(1987 ; 2000)$ en que la lógica con que estas empresas invirtieron en la industria automotriz estuvo 
guiada por la posibilidad de obtener parte de la ganancia que el sector podía realizar gracias al cierre de la importación de vehículos terminados. Baranson (1971, p. 30-31) ha observado que muchas empresas automotrices internacionales que invertían en países en desarrollo esperaban "obtener rápidos rendimientos durante los primeros dos a cuatro años, antes que las exigencias de fabricación local y la intensificación de las restricciones en materia de divisas empiecen a mermar los beneficios obtenidos en el país". No casualmente, varios de los proyectos fracasados no terminaron de ser expulsados del mercado cuando el gobierno exigió el cumplimiento de los compromisos de integración de partes locales, más caras que las importadas. Esta mayor exigencia hacia el sector quedó plasmada en el Decreto 6567/61 (ALA, tomo XXI, p. 738), complementario del 3693/59.

Junto con lo anterior, hay que destacar también el cambio de escenario que provocó la entrada de las "Tres Grandes" (Ford, Chrysler y General Motors) y de Fiat al mercado de automóviles no comerciales. Indudablemente, la intensificación de la competencia que esto trajo consigo y el establecimiento de una media de producción y de productividad más alta aceleraron la salida de los emprendimientos menores. ${ }^{8}$

Un segundo momento va desde 1961 a la crisis de 1963, cuando se produce una nueva ola de depuración del sector. De las 26 empresas originales, cuatro no produjeron nunca, cuatro más desaparecieron en los primeros dos años, y otras cinco se retiran o dejan de existir como fracción autónoma de capital en torno a la crisis. El resultado es elocuente: en cuatro años, la cantidad de empresas se redujo a la mitad. Al igual que en las sociedades capitalistas más industrializadas, la crisis aceleró las tendencias propias del movimiento de este modo de producción. ${ }^{9}$ Parecía confirmarse la validez de la política del desarrollismo de combinar un fuerte proteccionismo externo con una amplia libertad de mercado interna en pos de que la competencia seleccionara a los más aptos. ${ }^{10}$

8 Esta entrada afectó al conjunto del sector y no sólo a las empresas más pequeñas. Para ver la reacción de una empresa de la talla de SIAM ante este hecho, ver Rougier y Schvarzer, 2006, p. 31-32 (en particular la nota 14).

9 "La competencia prolifera aquí en razón directa al número y en razón inversa a la magnitud de los capitales rivales. Finaliza siempre con la ruina de muchos capitalistas menores, cuyos capitales en parte pasan a las manos del vencedor, en parte desaparecen" (Marx, 1995, p. 779, nota a $3^{\mathrm{a}} / 4^{\mathrm{a}}$ ed.).

10 "Establecido el principio de que el proteccionismo conviene al desarrollo económico del país, nosotros entendemos también que la libre competencia interna es fundamental para la expansión de las fuerzas productivas" (Luna, 1963, p. 79-80). 
Las primeras seis empresas produjeron cerca del $80 \%$ del total de la rama, mientras que ninguna de las firmas que se retiraron llegó a aportar siquiera el $1 \%$ de ese total. Si consideramos al conjunto de las nueve empresas que se vieron obligadas a retirarse resulta que su aporte fue de menos del 2,6\% durante el período (ver Cuadro 2).

\section{CUADRO 2}

Participación porcentual de las empresas en la producción anual, 1959-1964

\begin{tabular}{|l|c|c|c|c|c|c|c|}
\hline \multicolumn{1}{|c|}{ Empresa } & $\mathbf{1 9 5 9}$ & $\mathbf{1 9 6 0}$ & $\mathbf{1 9 6 1}$ & $\mathbf{1 9 6 2}$ & $\mathbf{1 9 6 3}$ & $\mathbf{1 9 6 4}$ & $\begin{array}{c}\mathbf{1 9 5 9} \\
\mathbf{1 9 6 4}\end{array}$ \\
\hline Industrias Kaiser Argentina S.A. & $\mathbf{7 3 , 7}$ & $\mathbf{3 7 , 3}$ & $\mathbf{3 0 , 9}$ & $\mathbf{3 0 , 8}$ & $\mathbf{2 6 , 4}$ & $\mathbf{3 0 , 2}$ & 33 \\
\hline Ford Motor Argentina S.A. & $\mathbf{2 , 2}$ & 13,2 & 9,9 & 9,1 & 8,6 & 16,3 & 11,2 \\
\hline Fiat Someca Concord S.A. & - & 4,8 & 8,3 & 10,9 & 17,7 & 14,2 & 10,9 \\
\hline General Motors Argentina S.A. & - & 12,4 & 9,9 & 9,3 & 8,7 & 11,6 & 9,9 \\
\hline Siam Di Tella Automotores S.A. & - & 4,6 & 10,3 & 5,5 & 8,1 & 7,0 & 6,9 \\
\hline $\begin{array}{l}\text { Févre y Basset Ltda., S.A. y } \\
\text { Chrysler Argentina S.A. }\end{array}$ & - & 4,9 & 5,4 & 7,7 & 7,8 & 6,3 & 6,1 \\
\hline IAFA S.A. (Peugeot) & - & 2,1 & 3,7 & 6,8 & 8,0 & 1,6 & 4,1 \\
\hline D.I.N.F.I.A. (ex I.A.M.E.) & 12,1 & 4,2 & 2,4 & 2,9 & 4,0 & 2,3 & 3,4 \\
\hline Citröen Argentina S.A. & - & 1,1 & 3,1 & 4,2 & 3,1 & 4,2 & 3,2 \\
\hline $\begin{array}{l}\text { Industria Automotriz Santa } \\
\text { Fe S.A. }\end{array}$ & - & 1,0 & 2,2 & 3,1 & 3,3 & 3,6 & 2,6 \\
\hline Isard Argentina S.A. & 2,8 & 1,7 & 2,9 & 3,5 & 2,2 & 1,4 & 2,4 \\
\hline Mercedes Benz Argentina S.A. & 2,4 & 2,9 & 2,7 & 1,8 & 1,6 & 1,3 & 2 \\
\hline Metalmecánica S.A. & 3,3 & 3,8 & 3,3 & 1,5 & 0,2 & 0,09 & 1,7 \\
\hline $\begin{array}{l}\text { Autoar Automotores } \\
\text { Argentinos S.A. }\end{array}$ & 0,7 & 1,6 & 1,8 & 1,3 & 0,2 & - & 0,9 \\
\hline Los cedros S.A. & 2,1 & 2,7 & 0,9 & 0,8 & - & - & 0,8 \\
\hline $\begin{array}{l}\text { Dinborg - Industrias Argentinas } \\
\text { Automotrices }\end{array}$ & - & 0,7 & 1,2 & 0,4 & - & - & 0,4 \\
\hline Goliath Hansa Argentina S.A. & - & 0,5 & 0,8 & - & - & - & 0,2 \\
\hline Cisitalia Argentina S.A. & - & - & 0,2 & 0,3 & - & - & 0,09 \\
\hline $\begin{array}{l}\text { Fábrica de Automotores } \\
\text { Utilitarios S.A. }\end{array}$ & 0,4 & 0,3 & - & - & - & - & 0,06 \\
\hline Panambí S.A. & 0,3 & 0,01 & - & - & - & - & 0,02 \\
\hline Onofre Marimón y Cía. SRL & - & 0,01 & 0,03 & - & - & - & 0,007 \\
\hline $\begin{array}{l}\text { Industrias de Transporte } \\
\text { Automotor S.A. }\end{array}$ & - & 0,1 & 0,1 & 0,1 & - & 0,08 \\
\hline
\end{tabular}

FUENTE: elaboración propia en base a datos de CONADE, 1966, cuadro 33. 
Por otra parte, del cruce de datos de ADEFA (1964b) sobre sus empresas asociadas ${ }^{11}$ con los valores totales recogidos por CONADE (1966) surge que las primeras ocuparon entre el 73 y el $85 \%$ del total del personal del sector (ver Cuadro 3) en el período 1959-1963.

\section{CUADRO 3}

Ocupación en la industria automotriz, 1959-1963

(números absolutos $\mathrm{y} \%$ )

\begin{tabular}{|c|c|c|c|c|c|c|}
\hline Años & $\begin{array}{c}\text { Personal } \\
\text { ocupado } \\
\text { ADEFA }\end{array}$ & $\%$ & $\begin{array}{c}\text { Personal } \\
\text { ocupado } \\
\text { por el resto }\end{array}$ & $\%$ & $\begin{array}{c}\text { Total de } \\
\text { personal } \\
\text { ocupado }\end{array}$ & $\%$ \\
\hline 1959 & 8.509 & 73 & 3.059 & 27 & 11.562 & 100 \\
\hline 1960 & 15.096 & 81 & 3.524 & 19 & 18.620 & 100 \\
\hline 1961 & 19.585 & 83 & 3.935 & 17 & 23.520 & 100 \\
\hline 1962 & 20.114 & 73 & 7.432 & 27 & 27.546 & 100 \\
\hline 1963 & 22.872 & 85 & 3.840 & 15 & 26.712 & 100 \\
\hline
\end{tabular}

FUENTE: elaboración propia con base en datos de ADEFA, 1964b, p. 3, y CONADE, 1966, cuadro 23.

\section{El desarrollo de la rama a nivel nacional e internacional}

Sin lugar a dudas, vista desde el rotundo fracaso de los emprendimientos menores, las empresas más grandes dentro del país aparecen como exitosas. Pero el panorama cambia radicalmente si la comparación la establecemos con los parámetros que regían en las empresas más importantes en el mercado mundial.

En primer lugar, si al analizar el Cuadro 2 remarcábamos una diferencia de escala notoria entre las primeras y últimas empresas en cuanto a la producción, podemos afirmar que la discrepancia entre la escala de la industria automotriz argentina y la imperante a nivel internacional es aún mayor. Ante todo, el parque de automóviles nacional fue entre 1959 y 1963 en porcentaje del 0,66; 0,68; 0,72; 0,76 y 0,78 del parque mundial (Coscia, 1980, cuadro II.5). En el plano productivo, mientras que la rama en Argentina durante el período considerado no llegó a superar las 200.000 unidades anuales divididas en una gran diversidad de

11 Las primeras seis del Cuadro 2 más Mercedes Benz. 
modelos, ${ }^{12}$ internacionalmente se calculaba que el volumen mínimo aceptable era de 250.000 unidades/anuales de un mismo modelo durante 6 a 8 años (C.E.E.I.A., 1969, p. 21). En contraste, las dos empresas automotrices más grandes del mundo, General Motors y Ford, ambas norteamericanas, produjeron en 1965 más de 8,8 millones de vehículos, cifra que representaba el $36,5 \%$ de la producción mundial.

Inevitablemente, esta realidad se reflejaba en los costos de producción. Visto el proceso desde el espacio nacional, y tal como lo ha señalado ADEFA, entre 1959 y 1964 los precios de los automóviles aunque aumentaron en términos nominales debido a la dinámica inflacionaria, lo hicieron en un $21,8 \%$ por debajo de los precios mayoristas del sector no agropecuario lo que resulta en una baja real de los precios del ramo (ADEFA, 1964, p. 5; cálculo a precios índice 1960). No obstante, ese abaratamiento no siguió el ritmo de los vehículos similares fabricados en los países de origen de las terminales, razón por la cual el costo local aumentó comparativamente a lo largo del tiempo (Coscia, 1980, 4.2). Por ejemplo, en 1967 el costo de un automóvil producido en Argentina era más de dos veces superior a uno similar realizado en los Estados Unidos (Baranson, 1971, p. 53 y 55; C.E.E.I.A., 1969, p. 180).

Esta información permite relativizar el énfasis que varios estudios han puesto en la alta concentración de la rama en la Argentina (Abot et al., 1974; Remes Lenicov, 1974). Resultaría más acertado reconocer que la tendencia a la centralización y depuración de capitales, propia del capitalismo en general, aunque opera localmente, lo hace de tal forma que continúa reproduciendo una escala pequeña de acumulación ya que aquí, empresas que producen menos de 50 mil unidades/anuales son las de mayor peso. En este sentido, es significativo que la fábrica de mayor producción en Argentina en el período bajo estudio haya sido IKA, cuando la Kaiser en su país de origen no había podido competir por sus costos excesivos, ni siquiera en el nicho del mercado de los autos pequeños en el que aún no habían entrado las "Tres Grandes" (White, 1972, p. 186).

Otra de las expresiones del desarrollo desigual de la rama se encuentra en las características del proceso de producción,

12 En 1960 se produjeron 50 modelos, considerando a ocho tipos de vehículos fabricados; en 1964 esa cantidad aumentó a 57 (Remes Lenicov, 1974, p. 106 y sigs.). Un año después, el total de modelos llegaba a 68 (Baranson, 1971, cuadro 12 anexo). 
especialmente en el nivel de predominio del factor objetivo sobre el subjetivo en el proceso de trabajo. La diferencia de tamaño - el de las plantas en Argentina no superaban el 10 o 15\% del considerado normal en los países más industrializados (Katz y Kosacoff, 1989, p. 54) - impedía la utilización de tecnología de punta lo que hacía que la calidad final dependiera de la fuerza de trabajo especializada en lugar de quedar garantizado por el propio proceso automático o mecanizado. Por ejemplo, Brennan (1996, p. 124-126) indica que en las automotrices instaladas en Córdoba la terminación de las matrices se hacía a mano, asimismo, "los bloques de motor seguían moviéndose manualmente, en pequeños carretones, más que en cintas transportadores o máquinas de transferencia, y los obreros trabajaban en ellos utilizando diversas máquinas herramienta de usos múltiples". En la filial de Fiat no contaban con los taladros automáticos que se empleaban Turín y en esta fábrica y en IKA el montaje del motor se hacía a mano. Baranson (1971, p. 58) refiere que en las automotrices en Argentina se usaba "un instrumental de soldadura portátil y remachadores manuales, en lugar del equipo ... automatizado que se utiliza en las cadenas de montaje de Detroit". Por otra parte, la complejidad de algunos de esos procesos obligó a las empresas terminales a operar con un nivel de integración vertical superior al que tenían en sus países de origen debido a las dificultades de conseguir esas partes en el mercado local.

\section{El desarrollo específico de la industria automotriz en la Argentina: su carácter desigual y combinado}

La instalación de las automotrices estuvo por lo tanto lejos de permitir superar la restricción externa y la dependencia tecnológica. Por el contrario, impulsó el drenaje de divisas no sólo a través de las importaciones sino que las remesas de utilidades y el pago de regalías pesaron cada vez más en el balance de pagos (Raddavero, 1972). ${ }^{13}$ Adicionalmente, la "brecha tecnológica" existente entre los países más industrializados y Argentina se amplió. Una de las consecuencias del desarrollo desigual de las fuerzas productivas, fue la imposibilidad de exportar al mercado mundial los

13 Este tipo de comportamiento caracterizó a todas las empresas, también a las "nacionales" (CONADE, 1966, anexo a). Los contratos de patentes, marca y asistencia fomentaban el flujo de importaciones desde la empresa licenciante, aun cuando esto no estuviera estipulado en el acuerdo escrito (Raddavero, 1972, p. 383). 
automóviles fabricados en este espacio nacional. Por lo tanto, la rama no sólo promovió un aumento de las importaciones y de las remesas, sino que no pudo contrapesar esa salida con ingresos derivados de la exportación. ${ }^{14}$

En definitiva, la forma técnica del proceso no hace más que revelar la especificidad de la Argentina como espacio de acumulación. Podemos sostener que el conjunto de determinaciones que dio forma al proceso en sus inicios se constituyó también en un resultado del mismo. Lejos de ser una diferencia meramente cuantitativa o técnica, el desarrollo desigual era un fenómeno más complejo. ${ }^{15}$ La cuestión más compleja consiste en resolver por qué las mismas empresas que se posicionaban en los primeros lugares de la producción mundial, con enormes magnitudes de adelanto de capital, de innovación tecnológica y de ventas, estuvieron interesadas en instalarse en un país como la Argentina.

Como ha sostenido Schvarzer ${ }^{16}$ la iniciativa de las transformaciones que se producen localmente se encuentra en las necesidades de las empresas transnacionales. La capacidad de estos grandes capitales para modificar las instituciones que ponen obstáculos a la internacionalización ha sido remarcada en repetidas ocasiones. Hay un hecho vinculado a ello que no aparece en la literatura y que muestra que los grandes capitales lejos de ser observadores pasivos de las políticas que toman los distintos gobiernos, promueven activamente las medidas que responden a sus intereses. Anteriormente nos hemos referido a los efectos que tuvo el ingreso de las "Tres Grandes" norteamericanas al país. Desde diciembre de 1959 Argentina había firmado con Estados

14 "Es inmediato de la observación de los datos - cualquiera sea su calificación - que el saldo directo en divisas de las operaciones del complejo [automotor] ha sido, sobre todo en los años iniciales, sustantivamente negativo" (Sourrouille, 1980, p. 187). "A partir del año 1959 ... comienzan nuevamente a aumentar las importaciones del sector Automotor"; “... para el período 1958-64 lo remesado en concepto de utilidades fue ya superior a lo realmente invertido" (Remes Lenicov, 1974, p. 129 y 132).

15 A pesar de discrepar con su repuesta a la cuestión, consideramos un verdadero aporte de J. Iñigo Carrera (1998) el haber planteado de un modo sistemático el problema teórico de la especificidad de la acumulación en Argentina.

16 "A pesar del interés de los inversores externos, ese proyecto se presentó como 'nacional' y se lo caracterizó como industrialización sustitutiva de importaciones; se lo podría haber llamado con más precisión, de 'industrialización por desborde de las empresas transnacionales de su mercado local'. La repetición de los mismos fenómenos en todas las grandes naciones de América Latina, donde actuaron las mismas transnacionales en las mismas ramas, sugiere que las políticas locales apenas tendieron a poner un 'toque' propio a un proceso global" (Schvarzer, 2000, p. 222, destacado nuestro). 
Unidos un acuerdo por el cual el gobierno norteamericano garantizaba el monto del capital radicado a los inversores que de esa nacionalidad que lo solicitaran en caso de que el gobierno argentino decretara la inconvertibilidad de su moneda. Para tener validez definitiva, el convenio debía ser ratificado por el Congreso. En el debate, el senador jujeño Benjamín Guzmán, sostuvo “tengo conocimiento personal que muchas compañías, entre ellas Ford y la General Motors, están esperando precisamente la ratificación de este convenio" (DSCS, 1\% /9/1960). El proyecto de ley fue aprobado en esa cámara; con un debate más agitado, la de Diputados le dio la sanción final a la Ley 15.803 en sesión extraordinaria el 25 de abril de $1961 .{ }^{17}$

Adicionalmente, la limitación de la mirada a un espacio nacional suele dejar en segundo plano lo que hay de general en procesos que son presentados como particulares. En este sentido, cabe recordarse que el proceso que llevó a la instalación de transnacionales automotrices en la Argentina se extendió a numerosos países. En julio de 1966 los países más industrializados (Estados Unidos, Gran Bretaña, Francia, Alemania Occidental, Japón, Italia y Suecia) tenían funcionando 387 cadenas de montaje en 55 países (Baranson, 1971, cuadro 6 anexo). La dimensión del proceso implica que esta tendencia no se originó en decisiones aisladas de las empresas.

Sin pretender dar una respuesta acabada a la pregunta de por qué las empresas transnacionales se instalaron en Argentina y por qué lo hicieron de ese modo - problema que seguimos investigando -, un aspecto fundamental a tomar en consideración es el momento que atravesaba el capitalismo, en particular en los países más industrializados. Luego de la inmediata posguerra, la recuperación de la producción automovilística europea permitió a las empresas de ese origen reposicionarse en el mercado mundial e incluso ingresar en el mercado norteamericano ofreciendo autos pequeños (White, 1972). La creciente competencia impulsó la aceleración de la innovación tecnológica - algunos autores la han denominado la "tercera revolución tecnológica" - avanzó en la automatización de los procesos productivos. La reducción del precio de los automóviles por medio de un impresionante aumento de la productividad, multiplicaba, a su vez, la competencia entre los

17 "Ratificación de un acuerdo con los Estados Unidos de Norte América sobre garantía de inversiones" (ALA, 1961, tomo XXIA, p. 15 y sigs.). 
distintos fabricantes que se veían compelidos a introducir innovaciones. El resultado fue una aceleración en el tiempo de rotación del capital fijo que se tradujo en períodos de amortización acortados y una obsolescencia más rápida (Mandel, 1979, cap. VIIVIII). Varias empresas internacionales del ramo se fusionaron y/o desparecieron entre la segunda mitad de la década del cincuenta y la siguiente por no poder seguir este ritmo. ${ }^{18}$ No casualmente, muchas de ellas fueron las licenciantes de patentes y marcas con las que firmaron contrato las empresas más chicas de la Argentina. ${ }^{19}$

Para las empresas más exitosas la dinámica que adquiría el proceso implicaba la desvalorización de maquinarias que, en caso de emplearla en el espacio de valor de su país de origen, habrían resultado en costos de producción por encima de la media. Las economías de los países "subdesarrollados" o "en vías de desarrollo" ofrecían la posibilidad no sólo de valorizar ese capital constante sino de obtener ganancias extraordinarias al realizar la producción dentro de los mercados internos de esos países gracias a que su nivel tecnológico aunque obsoleto en el país central, estaba por encima de la media en el país de destino.

La forma específica en que se desarrolló la industria automotriz en la Argentina en los años iniciales de su expansión es el resultado de un proceso en el que, citando a Schvarzer, "las políticas locales apenas tendieron a poner un 'toque' propio a un proceso global".

\section{Referencias}

Abot, Jorge; Abramzón, Mónica; Chorne, Miriam; Fariña, Eduardo; Khavisse, Miguel y Torre, Juan Carlos (1974). La concentración en la industria argentina en 1964. En: CONADE. El desarrollo industrial de la Argentina: sustitución de importaciones, concentración económica y capital extranjero (1950-1970). Argentina.

Altimir, Oscar, Santamaría, Horacio y Sourrouille, Juan (1966). Los instrumentos de la promoción industrial en la posguerra. Desarrollo Económico. Buenos Aires: IDES, v. 6, n. 21.

Anales de Legislación Argentina: 1958 (1959). Buenos Aires: Ediciones La Ley, tomo XVIIIA.

18 "En rigor de verdad, una de las principales razones por las que Kaiser había trasladado sus operaciones de los Estados Unidos a la Argentina era que no podía invertir en máquinas automatizadas" (Brennan, 1996, p. 125).

19 A modo de ejemplo: Borgward (licenciante de Dinborg) fue a la bancarrota en 1961; Goliath Werke, un desprendimiento de Borgward (licenciante de Goliath Hansa) sobrevivió sólo hasta 1963 cuando cerró; Hans Glass (licenciante de Isard) fue comprada por BMW hacia 1960. 
Anales de Legislación Argentina: 1959. Buenos Aires: Ediciones La Ley, tomo XIX A, 1960.

Anales de Legislación Argentina: 1961 (1962). Buenos Aires: Ediciones La Ley, tomo XXIA.

Asociación de Fábricas de Automotores (1964). La industria automotriz argentina. Su gravitación en el desarrollo del país. Buenos Aires.

Asociación de Fábricas de Automotores (1964b). Informe estadístico n. 133. Buenos Aires.

Baranson, Jack (1971). La industria automotriz en los países en desarrollo. Madrid: Editorial Tecnos - Banco Mundial.

Belini, Claudio (2006). Negocios, poder y política industrial en los orígenes de la industria automotriz argentina, 1943-1958. Revista de Historia Industrial. Barcelona: Publicacions i Edicions Universitat de Barcelona, n. 31, año XV.

Brennan, James (1996). El cordobazo: las guerras obreras en Córdoba, 1955-1976. Buenos Aires: Editorial Sudamericana.

Catalan, Jordi (2007). Protection and national systems of innovation: The takeoff of the automobile industry in Argentina, Spain and South Korea, 1945-87. En: 1er Congreso Latinoamericano de Historia Económica. Montevideo: 5-7 noviembre.

Comisión de Estudios Económicos de la Industria Automotriz (1969). La industria automotriz argentina. Informe económico. Argentina.

Consejo Nacional de Desarrollo (1963). Diagnóstico de la Economía Argentina 1953-1962. Buenos Aires.

Consejo Nacional de Desarrollo (1966). La industria automotriz: análisis preliminar. Buenos Aires.

Consejo Nacional de Desarrollo (1965). Plan de desarrollo 1965-1969. Buenos Aires.

Coscia, Santiago (1980). Evolución, dinámica actual y perspectivas de la industria automotriz. Argentina: Banco Nacional de Desarrollo, Gerencia de Investigaciones Económicas, Departamento de Estudios Económicos Sectoriales.

Diario de Sesiones de la Cámara de Senadores (1960). Sesiones ordinarias. Buenos Aires: Publicación del Cuerpo de Taquígrafos del Senado de la Nación.

Dorfman, Adolfo (1963). Cincuenta años de industrialización en la Argentina 1930-1980. Buenos Aires: Ed. Solar- Hachette.

Instituto Nacional de Estadística y Censos. Censo Nacional Económico 1963 (1965). Industria manufacturera: resultados generales relevado el 30 de abril de 1964 Informe Serie C.N.E.2. Buenos Aires: INDEC.

Iñigo Carrera, Juan (1998). La acumulación de capital en la Argentina. Argentina: Centro para la Investigación como Práctica Política.

Jerez, Patricia (2007). Treinta años en la evolución de la industria siderúrgica argentina (1946-1976). Una cuestión de oferta y demanda. En: Rougier, Marcelo (dir.). Políticas de promoción y estrategias empresariales en la industria argentina. 1950-1980. Buenos Aires: Ediciones Cooperativas.

Katz, Jorge y Kosacoff, Bernardo (1989). El proceso de industrialización en la Argentina: evolución, retroceso y prospectiva. Buenos Aires: Centro Editor de América Latina - CEPAL. 
Luna, Félix (1979). Diálogos con Frondizi. Buenos Aires: Editorial Desarrollo. Mandel, Ernest (1979). El Capitalismo Tardío. México: Ediciones Era.

Marx, Karl (1995). El Capital. Crítica de la Economía Política. España - México: Siglo XXI, t. 1, v. 3.

Raddavero, Bruno C. (1972). Análisis de la transferencia de la tecnología externa a la industria argentina: el caso de la industria automotriz. Económica, La Plata, Argentina, año XVIII, n. 3.

Remes Lenicov, Jorge L. (1974). Algunos resultados de la política desarrollista (1958-1964): el caso de la industria automotriz. En: Consejo Profesional de Ciencias Económicas de la Provincia de Buenos Aires, Universidad Nacional de La Plata. Facultad de Ciencias Económicas. Problemas económicos argentinos: diagnóstico y políticas. Buenos Aires: Ediciones Macchi.

Rougier, Marcelo y Schvarzer, Jorge (2006). Las grandes empresas no mueren de pie. El (o)caso de SIAM. Buenos Aires: Grupo Editorial Norma.

Schvarzer, Jorge (2000). La industria que supimos conseguir. Buenos Aires: Ediciones Cooperativas.

Schvarzer, Jorge (1995). La reconversión de la industria automotriz argentina: un balance a mitad de camino. Ciclos, Buenos Aires, año V, v. V, n. 8.

Schvarzer, Jorge (1977). Las empresas industriales más grandes de la Argentina. Una evaluación. Desarrollo Económico, Buenos Aires: IDES, v. 17, n. 66.

Schvarzer, Jorge (1987). Promoción industrial en Argentina. Características, evolución y resultados. Buenos Aires: CISEA.

Sourrouille, Juan Vital (1980). Transnacionales en América Latina. El complejo automotor en Argentina. México: ILET - Editorial Nueva Imagen.

White, Lawrence (1972). The American automobile industry and the small car, 1945-1970. The Journal of Industrial Economics, v. 20, n. 2. 